\title{
Control of ventricular unloading using an electrocardiogram-synchronized Thoratec paracorporeal ventricular assist device
}

\author{
Raffael Amacher, MSc, ${ }^{\mathrm{a}}$ Alberto Weber, MD, ${ }^{\mathrm{b}}$ Henriette Brinks, MD, ${ }^{\mathrm{b}}$ Shannon Axiak, DVM, ${ }^{\mathrm{c}}$ \\ Antonio Ferreira, PhD, ${ }^{\mathrm{d}}$ Lino Guzzella, PhD, ${ }^{\mathrm{a}}$ Thierry Carrel, MD, ${ }^{\mathrm{b}}$ James Antaki, PhD, ${ }^{\mathrm{e}}$ and \\ Stijn Vandenberghe, $\mathrm{PhD}^{\mathrm{f}}$
}

\begin{abstract}
Objective: Current pulsatile ventricular assist devices operate asynchronous with the left ventricle in fixed-rate or fill-to-empty modes because electrocardiogram-triggered modes have been abandoned. We hypothesize that varying the ejection delay in the synchronized mode yields more precise control of hemodynamics and left ventricular loading. This allows for a refined management that may be clinically beneficial.
\end{abstract}

\begin{abstract}
Methods: Eight sheep received a Thoratec paracorporeal ventricular assist device (Thoratec Corp, Pleasanton, Calif) via ventriculo-aortic cannulation. Left ventricular pressure and volume, aortic pressure, pulmonary flow, pump chamber pressure, and pump inflow and outflow were recorded. The pump was driven by a clinical pneumatic drive unit (Medos Medizintechnik AG, Stolberg, Germany) synchronously with the native R-wave. The start of pump ejection was delayed between $0 \%$ and $100 \%$ of the cardiac period in $10 \%$ increments. For each of these delays, hemodynamic variables were compared with baseline data using paired $t$ tests.
\end{abstract}

Results: The location of the minimum of stroke work was observed at a delay of $10 \%$ (soon after aortic valve opening), resulting in a median of $43 \%$ reduction in stroke work compared with baseline. Maximum stroke work occurred at a median delay of $70 \%$ with a median stroke work increase of $11 \%$ above baseline. Left ventricular volume unloading expressed by end-diastolic volume was most pronounced for copulsation (delay $0 \%$ ).

Conclusions: The timing of pump ejection in synchronized mode yields control over left ventricular energetics and can be a method to achieve gradual reloading of a recoverable left ventricle. The traditionally suggested counterpulsation is not optimal in ventriculo-aortic cannulation when maximum unloading is desired. (J Thorac Cardiovasc Surg 2013;146:710-7)

In selected patients with advanced heart failure, the use of left ventricular assist devices (LVADs) has resulted in prolonged survival and a better quality of life. ${ }^{1,2}$ Over the past decade, the design of LVADs intended for long-term use has evolved from the first generation of volumedisplacement, or pulsatile, pumps to today's smaller and lighter continuous-flow pumps. ${ }^{3,4}$ The latter have obvious benefits, the major one being the possibility of intrathoracic implantation. In addition, the newer devices

From the Institute for Dynamic Systems and Control, ${ }^{\mathrm{a}}$ ETH Zurich, Zurich, Switzerland; Department of Cardiovascular Surgery, ${ }^{\mathrm{b}}$ Inselspital Bern, Bern, Switzerland; Department of Veterinary Anesthesia, ${ }^{\mathrm{c}}$ University of Bern, Bern, Switzerland; Department of Mathematics, ${ }^{\mathrm{d}}$ Federal University of Maranhao, Maranhao, Brazil, and Department of Biomedical Informatics, University of Pittsburgh, Pittsburgh, $\mathrm{Pa}$; Department of Biomedical Engineering, ${ }^{e}$ Carnegie Mellon University, Pittsburgh, Pa; and ARTORG Cardiovascular Engineering, ${ }^{\mathrm{f}}$ University of Bern, Bern, Switzerland

Funding: This study was supported by the Swiss National Science Foundation, Grant 32003B_127443. This study was supported in part by 1R01 HL086918.

Disclosures: Authors have nothing to disclose with regard to commercial support.

Received for publication Sept 27, 2012; revisions received Nov 15, 2012; accepted for publication Dec 11, 2012; available ahead of print Jan 14, 2013.

Address for reprints: Raffael Amacher, MSc, Institute for Dynamic Systems and Control, ETH Zürich, ML K36.3, Sonneggstrasse 3, 8092 Zürich, Switzerland (E-mail: raffael.amacher@idsc.mavt.ethz.ch).

0022-5223/\$36.00

Copyright (c) 2013 by The American Association for Thoracic Surgery http://dx.doi.org/10.1016/j.jtcvs.2012.12.048 with continuous flow may have a better long-term durability and a greater patient/physician acceptability because of their size, silent operation, and easier handling. However, their efficacy of left ventricular unloading and right ventricular afterload reduction may not be as pronounced as in the more physiologic pulsatile LVADs. ${ }^{5-7}$ Additional benefits of pulsatile ventricular assist devices (VADs) over continuousflow systems are the greater likelihood of aortic valve opening $^{6}$ and lower rate of gastrointestinal bleeding events. ${ }^{8}$

With both types of devices, it has been shown that ventricular reverse remodeling can be induced by mechanical circulatory support. ${ }^{9}$ However, it is unclear what degree and duration of ventricular unloading would optimize left ventricular recovery. Partial loading of the left ventricle (LV) by reducing the VAD rate ${ }^{10}$ or stroke volume,${ }^{11}$ for example, could be used for weaning patients from a VAD, given that the supported LV has the potential to gradually recover.

In terms of VAD cycle duration, there are 3 common operating modes of a pulsatile VAD: the fixed rate, synchronized to the heart rate, or the automatic mode wherein the VAD ejection is triggered when the pump chamber is full (recognized by a hall-effect switch). ${ }^{12}$ The latter has become the standard operating mode for pulsatile VADs once the early postoperative period has passed. However, 


\section{Abbreviations and Acronyms \\ $\mathrm{ECG}=$ electrocardiogram \\ $\mathrm{LV}=$ left ventricle \\ $\mathrm{LVAD}=$ left ventricular assist device \\ $\mathrm{VAD}=$ ventricular assist device}

the lack of synchrony between heart and pulsatile VAD causes a fluctuation in LV load that may impede recovery and even cause intermittent overloading. ${ }^{13}$

Therefore, we hypothesize that a synchronous mode of operation in combination with a prescribed phase shift (ejection delay relative to the RR duration) might be used for stabilizing and controlling the load on the recovering LV while maintaining perfusion. Such a control scheme would permit gradual "reloading therapy" and may have a beneficial effect on recovery rates. Although previous investigations in this matter are inconclusive, ${ }^{6,14-16}$ there has yet to be a clinical study in which the degree of ventricular reloading has been consistently, systematically maintained. An optimized pulsatile VAD actuation scheme as described would enable such a study to be performed.

In this study, we established a sheep model with pulsatile mechanical circulatory support in a synchronized mode to investigate the influence of phase shift on LV unloading described by pressure, volume, stroke work, and wall stress. A further aim of this study was to identify an optimal phase shift in which maximal ventricular unloading is achieved and to identify the contraindicated phase shift that risks cardiac overload.

\section{MATERIALS AND METHODS \\ Animals}

This experiment was approved by the Commission of Animal Experimentation of the Canton of Bern, Switzerland (Approval No. 52/09). A paracorporeal ventricular assist device (Thoratec Corp, Pleasanton, Calif) was implanted in 8 adult, female, random-bred sheep $(59-83 \mathrm{~kg})$.

\section{Anesthetic Management}

All animals were premedicated intramuscularly with $0.2 \mathrm{mg} / \mathrm{kg}$ of both midazolam and methadone. General anesthesia was induced with intravenous midazolam $(0.2 \mathrm{mg} / \mathrm{kg})$, ketamine $(3.5 \mathrm{mg} / \mathrm{kg})$, and propofol $(1-3 \mathrm{mg} / \mathrm{kg})$. After orotracheal intubation, anesthesia was maintained with isoflurane in oxygen (end-tidal concentration 1.6\%), fentanyl $(5 \mu \mathrm{g} / \mathrm{kg}$ bolus and then $5-10 \mu \mathrm{g} / \mathrm{kg} / \mathrm{h}$ intravenously), and rocuronium (0.15-0.6 mg/kg intravenously). The sheep were mechanically ventilated. An orogastric tube was placed to decompress the rumen, and the rectal temperature was maintained between $36^{\circ} \mathrm{C}$ and $38^{\circ} \mathrm{C}$ with a circulating warm air blanket. Severe intraoperative blood loss was compensated for by autologous blood transfusion with a cell saver (AutoLog; Medtronic Inc, Minneapolis, Minn). At the end of the experiment, the animals were euthanized under anesthesia with a lethal dose of intravenous potassium chloride.

\section{Surgical Preparation}

The pulsatile VAD was implanted via left lateral thoracotomy in the third intercostal space. A dual-pressure $7 \mathrm{~F}$ admittance catheter (Scisense Inc,
London, Ontario, Canada) was placed in the LV via the right carotid artery for measuring LV pressure, LV volume, and aortic pressure. An ultrasonic transit time flow probe (22PAU or 24PAU; Transonic Systems Inc, Ithaca, $\mathrm{NY}$ ) was placed around the pulmonary artery to measure total cardiac output. For inflow cannulation, we inserted a modified curved $32 \mathrm{~F}$ venous cannula (DLP67532, Medtronic Inc) into the left ventricular apex and stabilized it with a double-pledged purse-string suture. A 1.27 -cm polyvinyl chloride tube was extended with a 12-mm Biplex graft (Gelweave; Vascutek Terumo GmbH, Renfrewshire, Scotland) to form the outflow cannula, which was cut to length during surgery. The graft was anastomosed end to side to the proximal descending aorta because the ascending aorta cannot be approached safely from a lateral incision in the sheep and the pulmonary artery flow probe (mentioned earlier) interferes with a safe manipulation of the ascending aorta. The pump was mounted in a holder by the processus xiphoideus to allow for cannula lengths as in human cases. Ultrasonic flow probes (11PXL, Transonic Systems Inc) were installed on the cannulas for measuring pulsatile VAD inflow and outflow. Fluid-filled pressure transducers (Truwave; Edwards Lifesciences LLC, Irvine, Calif) were used to measure VAD chamber pressure, driveline air pressure, and left atrial pressure. To measure VAD chamber pressure, a luer connector was installed between the pump sac and the outflow valve. All pressure measurement devices were zeroed at atmosphere and calibrated with a handheld piston calibrator (PXCAL, Edwards Lifesciences LLC).

\section{Ventricular Assist Device Actuation}

The pulsatile VAD was actuated using a clinical pneumatic drive unit (Medos Medizintechnik AG, Stolberg, Germany) in external trigger mode. To improve the quality of synchronization, an application-specific algorithm was programmed in LabView (National Instruments, Austin, Tex) and run in real-time on a cRIO-9074 (National Instruments) to perform R-wave detection. ${ }^{17}$ This algorithm was extended with arrhythmia detection such that the sensitivity of the VAD rate to disturbances could be reduced. An analog signal to trigger VAD ejection was generated and applied to the external input of the clinical pneumatic drive unit.

The systolic and diastolic pressures were set at the beginning of each experiment to achieve maximum VAD outflow with a $50 \%$ phase shift and were further kept constant. The primary independent variable was the phase shift, which is the delay between the R-wave and the onset of the VAD systole, designated as the percentage of the actual RR duration (thereby responding dynamically to changes in the heart rate). This definition is illustrated in Figure 1, C. For all experiments, the systolic duration of the VAD was maintained at $35 \%$ of the current VAD cycle duration.

\section{Measurement Protocol}

At the beginning and the end of each experiment, a baseline recording was made with the VAD off and clamped. The pulsatile VAD was then synchronized to the heartbeat using the clinical pneumatic drive unit and the synchronization algorithm described earlier. In each experiment, 10 different phase shifts, equally spaced between $0 \%$ and $100 \%$, were applied for 5 minutes each. This allowed the analysis of the full range of phase shift settings, such that situations between the 2 extremes of co- and counterpulsation could be studied. The order of phase shift settings was randomized before the study and different for each animal.

\section{Pharmacologic Interventions}

In all animals, the same measurement protocol was repeated 3 times under different pharmacologic conditions. This allows analyzing the heartVAD interaction over a wider range of hemodynamic states such that the effects observed in our data are not limited to normal hemodynamic conditions. The first protocol involved baseline normotensive hemodynamics, described earlier. For the second protocol, phenylepherine (bolus, 1.25$2.25 \mu \mathrm{g} / \mathrm{kg}$; maintenance, $0.3-0.5 \mu \mathrm{g} / \mathrm{kg} / \mathrm{min}$ ) was used to increase afterload. Cardiac contractility was reduced in the third protocol using esmolol (initial bolus, $50 \mu \mathrm{g} / \mathrm{kg}$; maintenance, $5-20 \mu \mathrm{g} / \mathrm{kg} / \mathrm{min}$ ). The esmolol 

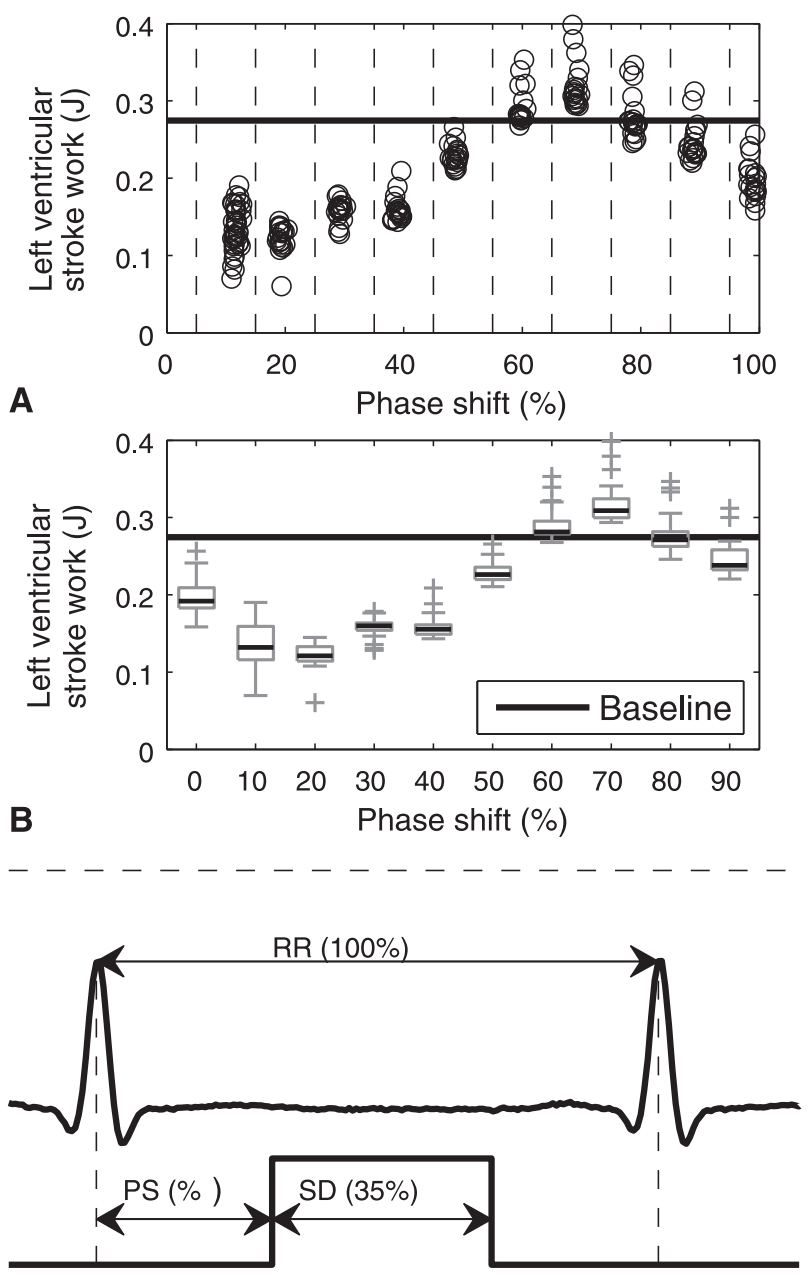

C

FIGURE 1. A and B, The procedure of data preparation for statistical analysis is illustrated for the example of stroke work in animal number 8 , proto$\operatorname{col} 3$ (decreased contractility). The beat-to-beat variables are assigned to 1 of 10 phase shift groups according to the actual phase shift. Group 0: $0 \%$ to $5 \%$ and $95 \%$ to $100 \%$. Group $1: 5 \%$ to $15 \%$. Group $2: 15 \%$ to $25 \%$. Group 3: $25 \%$ to $35 \%$. Group $4: 35 \%$ to $45 \%$. Group $5: 45 \%$ to $55 \%$. Group $6: 55 \%$ to $65 \%$. Group 7: $65 \%$ to $75 \%$. Group $8: 75 \%$ to $85 \%$. Group 9: $85 \%$ to $95 \%$. C, The definition of phase shift with respect to the RR duration is illustrated. The systolic duration of the pulsatile VAD is chosen to be constant $(35 \%)$. PS, Phase shift; RR, RR duration; $S D$, systolic duration.

dosage was then titrated upward every 5 minutes until the desired effect was achieved.

\section{Data Processing and Statistical Analysis}

Measured data were recorded using a digital acquisition system (400 Series, iWorx Systems Inc, Dover, NH). The recorded data were processed in MATLAB (R2011b, The Mathworks Inc, Natick, Mass). When steadystate hemodynamic conditions were achieved, the right ventricular cardiac output as measured at the pulmonary artery was used as a measure of total cardiac output. With the measured pulsatile VAD outflow, it was possible to obtain an estimate of the resulting volume flow through the aortic valve. The ratio of pulsatile VAD flow to total cardiac output was defined as the assistance ratio. Regurgitation from the pulsatile VAD to the LV was calculated as the negative portion of the flow probe data at the inlet cannula.
LV volume as measured by admittance was calibrated in post-processing by matching stroke volume from the catheter to that derived from the flow signal on the pulmonary artery (with deactivated device and clamped cannulas). Blood conductivity was measured after each protocol and included in the volume calculation according to Wei and colleagues' formula. ${ }^{18} \mathrm{LV}$ stroke work was calculated for each beat as the area in the pressure-volume plane. LV wall-stress was calculated using an ellipsoidal approximation described previously. ${ }^{19}$ The LV wall volume required for this method was obtained by excising the heart after euthanasia, removing the right ventricle and atria, and submerging the remaining $\mathrm{LV}$ in a graduated cylinder.

An interval of 20 heartbeats without arrhythmia events was selected for each phase shift setting for further analysis. For all the data sections chosen, the median values were used for further analysis to minimize the variation induced by breathing effects. These values were then assigned to 1 of 10 phase shift groups. This procedure is illustrated in Figure 1, $A$ and $B$.

Statistical analyses were performed using IBM SPSS Statistics 19 (SPSS Inc, Chicago, Ill). To compare data from a specific phase shift setting with baseline values, paired $t$ tests were used. The significance level used for all statistical tests performed was $5 \%$.

\section{RESULTS}

Eight of 24 recorded data sets were excluded from this study, leaving a total of 16 data sets from 6 animals available for further analysis. These data sets resulted from animals that remained in stable condition throughout the experiment. The 2 reasons for exclusion were strongly arrhythmic behavior and untrustworthy LV pressure recordings (catheter tip touches LV wall).

Pharmacologic interventions resulted in repeatable conditions over the 3 different protocols applied (standard, increased afterload, and decreased contractility), as illustrated in Figure 2. Mean stroke work for the 3 protocols was $0.39 \mathrm{~J}$ (standard deviation, $0.09 \mathrm{~J}), 0.5 \mathrm{~J}(0.13 \mathrm{~J})$, and $0.25 \mathrm{~J}(0.08$ $\mathrm{J})$, respectively. Corresponding values of mean arterial pressure were $57 \mathrm{~mm} \mathrm{Hg} \mathrm{(4} \mathrm{mm} \mathrm{Hg),} 77 \mathrm{~mm} \mathrm{Hg}(9 \mathrm{~mm} \mathrm{Hg})$, and $50 \mathrm{~mm} \mathrm{Hg}(6 \mathrm{~mm} \mathrm{Hg})$, respectively. These values represent the physiologic response expected from the interventions applied. The variation of heart rate (mean, 94 beats/min; range, $84-116$ beats/min), mean arterial pressure (mean, $58 \mathrm{~mm} \mathrm{Hg}$; range, 40-89 $\mathrm{mm} \mathrm{Hg}$ ), and cardiac output (mean, 5.3 L/min; range, $2.7-8.3 \mathrm{~L} / \mathrm{min}$ ) provided a wide range of hemodynamic conditions for consideration.

\section{Influence of Phase Shift on Left Ventricular Volume}

The phase shift was found to significantly influence the LV volume (Figures 3 and 4). In the pressure-volume plane, the isovolumic contraction and relaxation of the unsupported ventricle (in the absence of valve leakage) are represented by vertical lines. However, in our supported sheep hearts these phases can deviate significantly from the isovolumic line and tilt leftward or rightward depending on the phase shift. Regurgitation from the pump to the LV was $1.01 \mathrm{~L} / \mathrm{min}$ (mean flow over all data analyzed) and depended on the phase shift as shown in Figure 5, D, with the highest regurgitation at phase shifts $50 \%$ to $70 \%$. End-diastolic volume was significantly reduced with 

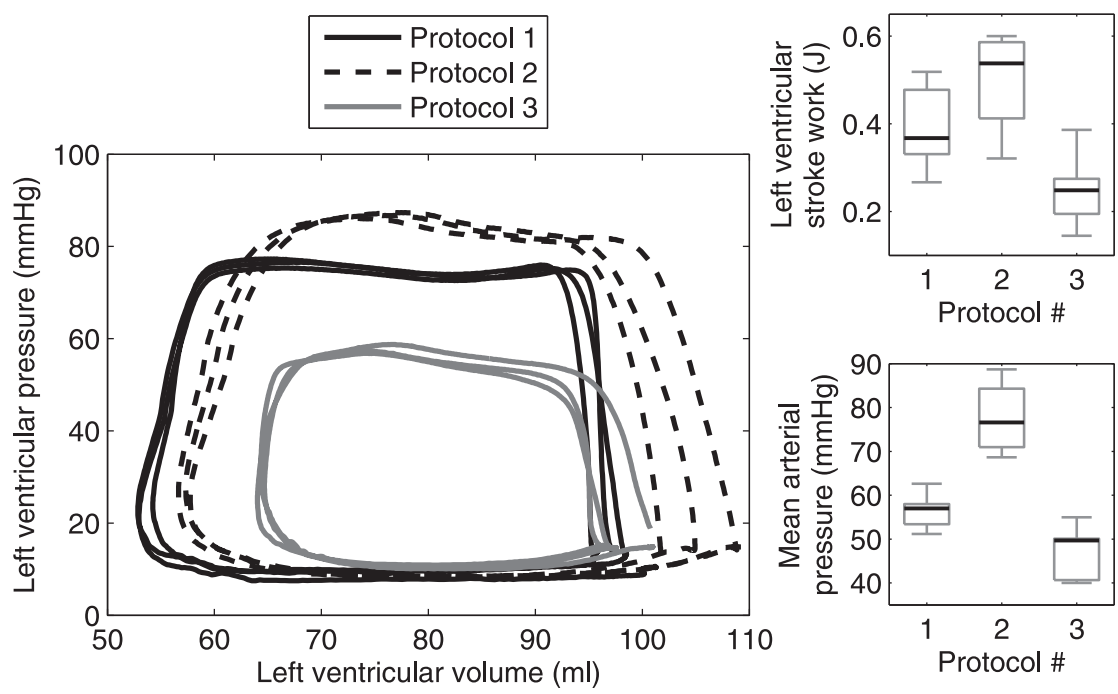

FIGURE 2. The left plot shows a representative example of LV pressure-volume loops in baseline (VAD off and clamped) from animal number 2 for the 3 different pharmacologic protocols applied (standard, increased afterload, and decreased contractility). For each of these cases, pressure-volume loops from 3 consecutive heartbeats are shown. On the right side, 2 boxplots show the response of LV stroke work and mean arterial pressure in baseline to the pharmacologic interventions.

respect to baseline for each phase shift setting (Figure 4,C). The minimum end-diastolic volume was observed at copulsation (phase shift $0 \%$ ).

\section{Influence of Phase Shift on Pressures}

The developed LV pressure was found to significantly depend on the phase shift (Figures 3 and 4, G). For the phase shifts $0 \%, 10 \%, 20 \%, 70 \%, 80 \%$, and $90 \%$, an increase of peak LV pressure above baseline was observed. Contrarily, peak LV pressure was low when the VAD diastole occurred during the LV systole (phase shifts $30 \%, 40 \%, 50 \%$, and $60 \%$ ). Mean left atrial pressure was significantly reduced compared with baseline except for the phase shift $40 \%$ (Figure $4, J$ ). Mean arterial pressure was increased over baseline for the phase shifts $0 \%, 10 \%, 20 \%, 30 \%, 80 \%$, and $90 \%$ (Figure $4, H$ ).

\section{Influence of Phase Shift on Left Ventricular Unloading}

We identified the phase shifts $0 \%$ to $50 \%$ to have a significant impact on unloading the LV in terms of stroke work (Figure $4, B$ ). The phase shift $10 \%$ had the greatest reduction in median stroke work $(-43 \%)$. Despite the fact that the median value is minimal for the phase shift $10 \%$, it was possible that the stroke work was greater than at baseline for individual cases in this phase shift.

Mean wall stress (Figure 4,D) was reduced compared with baseline for each phase shift setting except for the phase shifts $0 \%, 80 \%$, and $90 \%$. Peak wall stress (Figure $4, F$ ) was significantly greater than at baseline for the phase shifts $80 \%$ and $90 \%$.

\section{DISCUSSION}

We analyzed the complex interaction between a pulsatile VAD and the LV for synchronized mechanical circulatory support with different phase shifts (relative ejection delays) to obtain more knowledge about the relationship between pump timing and cardiac loading conditions in terms of volumes, pressures, stroke work, and wall stress. In the data presented, phase shift has been actively set at a desired value using a clinical pneumatic drive unit extended with custom-made software for improved electrocardiogram (ECG) triggering. Ten different phase shift settings have been applied. This allowed a detailed analysis of the resulting hemodynamics.

Actuating pulsatile VADs requires a number of parameters that have to be set by trained personnel during assistance, such as the rate, systolic duration, and filling and ejection pressures. ${ }^{12,20}$ Earlier publications studied the influence of selected choices for these parameters on the coupled pulsatile VAD-cardiovascular system in silico ${ }^{21,22}$ or in vivo. ${ }^{16,23}$ Most previous research has focused on the case of atrio-aortic cannulation, but in the clinical setting, ventriculo-aortic cannulation has now been established for the majority of LVADs. For the atrio-aortic configuration, it was concluded earlier that VAD ejection should occur soon after aortic valve closure to minimize the oxygen consumption of the LV. ${ }^{21,22}$ This unloading effect was obtained by LV preload reduction. For the atrio-aortic cannulation, the $\mathrm{LV}$ is not connected directly to the VAD, and therefore the afterload is exclusively determined by the aortic pressure. For ventriculo-aortic cannulation, however, the LV can eject in both the aorta and the pulsatile VAD. The afterload imposed on the LV thus depends on instantaneous 


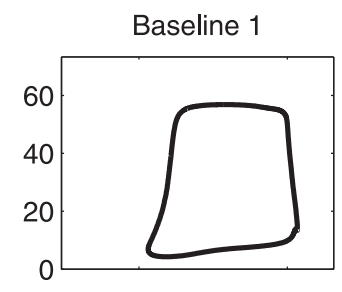

Phase shift: $29 \%$

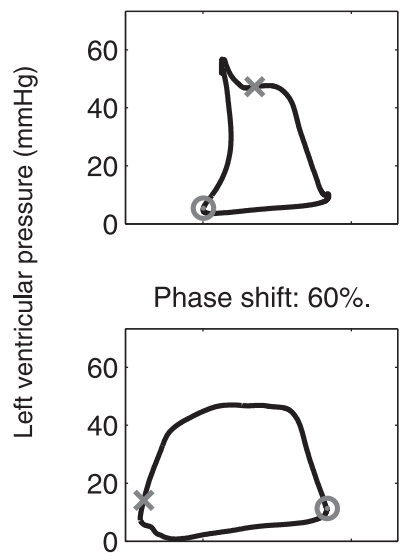

Phase shift: $89 \%$

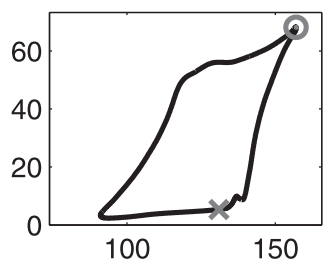

Phase shift: $12 \%$

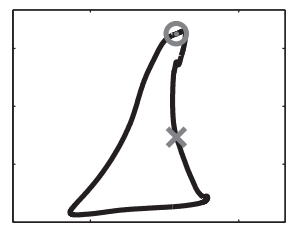

Phase shift: $39 \%$.

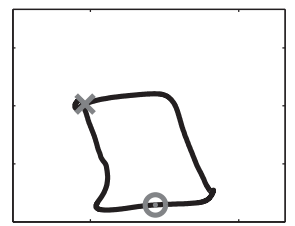

Phase shift: $69 \%$.

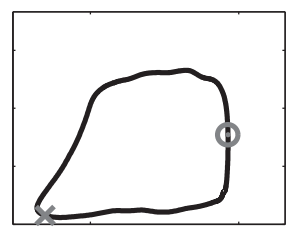

Phase shift: $99 \%$.

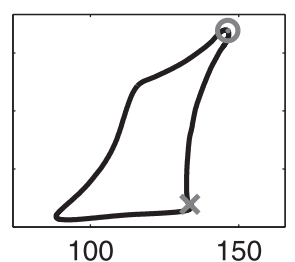

Phase shift: $19 \%$.

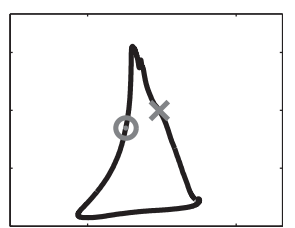

Phase shift: $49 \%$.

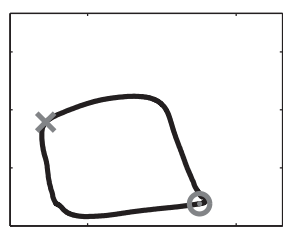

Phase shift: $79 \%$.

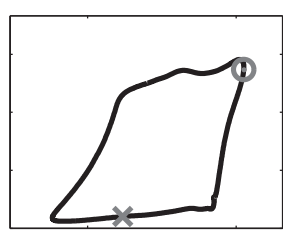

Baseline 2

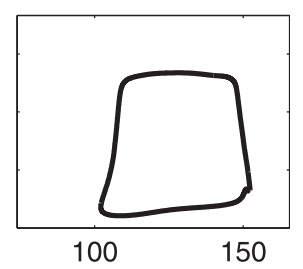

Left ventricular volume $(\mathrm{ml})$

FIGURE 3. LV pressure-volume loops for animal number 8, protocol 3 (decreased contractility). Baseline 1 and 2 are recorded immediately before and after applying the different VAD settings. The PV loops for the different phase shifts are ordered by increasing phase shifts from top left to bottom right. The symbol $\mathrm{x}$ denotes the start of VAD systole; o denotes the start of pump filling.

pump chamber pressure, and on timing of VAD ejection, as analyzed in this study.

Figure 3 shows that both the start and the end of pump ejection influence the shape of the LV pressure-volume loops. It can be seen that at phase shifts $39 \%$ and $49 \%$, the start of VAD systole stops blood ejection from the LV, whereas at phase shifts $79 \%, 89 \%$, and $99 \%$ ejection of blood from the LV starts immediately after the VAD systole is over. Thus, in addition to the start of pump ejection relative to the cardiac cycle, the end of pump ejection influences $\mathrm{LV}$ pressure and volume.

The phase shift in ventriculo-aortic cannulation influences not only the LV pressure developed but also the fraction of blood that is ejected from the LV through the aortic valve with respect to total LV stroke volume. Because of the controlled phase shift, different scenarios can be achieved.

For instance, the LV stroke volume and end-diastolic volume can be maximized for phase shifts between $50 \%$ and
$70 \%$, in which case most of the volume ejected from the LV contributes to pulsatile VAD filling.

In another case, the end-diastolic volume can be reduced maximally by using co-pulsation (phase shift $0 \%$ ) because in that case pump filling occurs during LV diastole and blood can flow through the LV into the pump chamber without expanding the LV. In this case, opening of the aortic valve is favored. This would help to prevent cusp fusion or thrombus formation at the level of the aortic valve.

The pulsatile VAD used contains tilting disc valves such that moderate regurgitation over both valves cannot be avoided completely. Previous authors have stated that pulsatile VAD inflow regurgitation could inhibit the ventricular reverse remodeling processes. ${ }^{24}$ The amount of regurgitation depends on the pressure difference across the valve and thus on the phase shift (Figure 5, D). By applying our method, it would be possible to minimize regurgitation by 

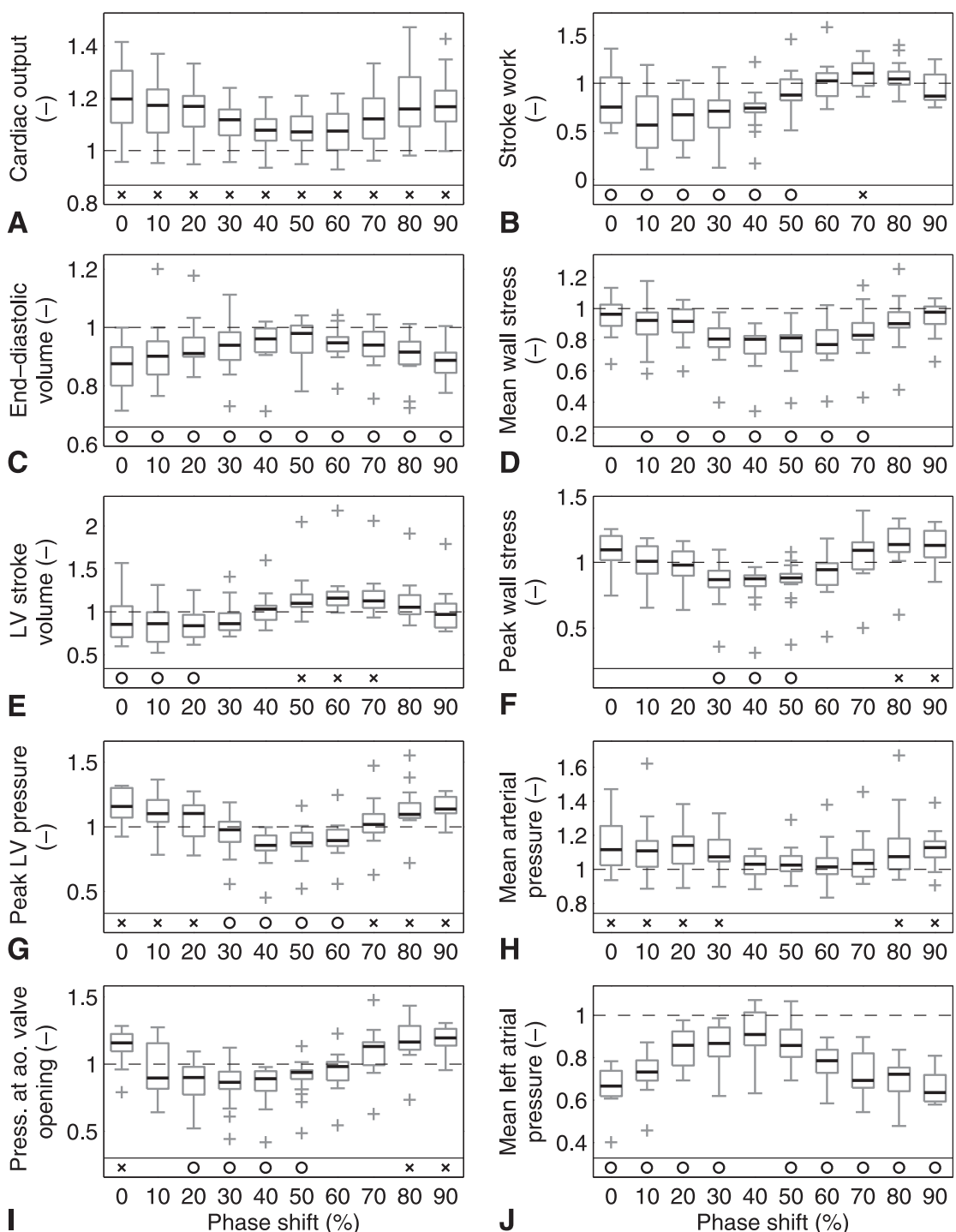

FIGURE 4. Boxplots of analyzed variables clustered according to their phase shift are shown (each box represents the median value and 25th and 75th percentiles; + denotes outliers). Data points are normalized by the baseline values of the individual data sets. Panel A through J show cardiac output, stroke work, end-diastolic volume, mean wall stress, LV stroke volume, peak wall stress, peak LV pressure, mean arterial pressure, LV pressure at aortic valve opening, and mean left atrial pressure. The outcome of the paired $t$ test is shown below the corresponding data (o: significant decrease compared with baseline, $\mathrm{x}$ : significant increase compared with baseline, no symbol: no significant difference compared with baseline). Mean left atrial pressure was available for only 8 different data sets. $L V$, Left ventricle.

applying a phase shift that leads to overlapping systolic phases of the pulsatile VAD and LV.

The results presented in terms of LV pressure agree with recently published data where a pulsatile VAD was implanted in a pig model. ${ }^{15}$ Both studies show that peak LV pressure was minimized for counterpulsation (phase shifts $30 \%$ to $60 \%$; Figure $4, G$ ). This phenomenon can be explained by the pulsatile VAD being in its filling phase during LV systole, and thus the LV can dump blood in the pump chamber without developing high pressure. Nevertheless, our results add knowledge to these findings because we also show that minimal LV pressure does not correspond with minimal LV load in terms of LV stroke work.
As is known from intra-aortic balloon counterpulsation, the end-diastolic arterial pressure, or pressure at aortic valve opening, is an important determinant of cardiac load. Our results (Figure $4, I$ ) indicate that this pressure can be minimized by a phase shift between $20 \%$ and $50 \%$.

As opposed to previous work, ${ }^{15}$ our study design enabled the analysis of myocardial wall stress. To achieve maximal unloading, a VAD should be operated such that LV wall stress is reduced. For the phase shifts $0 \%, 80 \%$, and $90 \%$, mean wall stress cannot be reduced compared with baseline; in addition, the phase shifts $80 \%$ and $90 \%$ lead to significantly elevated peak wall stress compared with baseline. These settings should be avoided in patients 
A
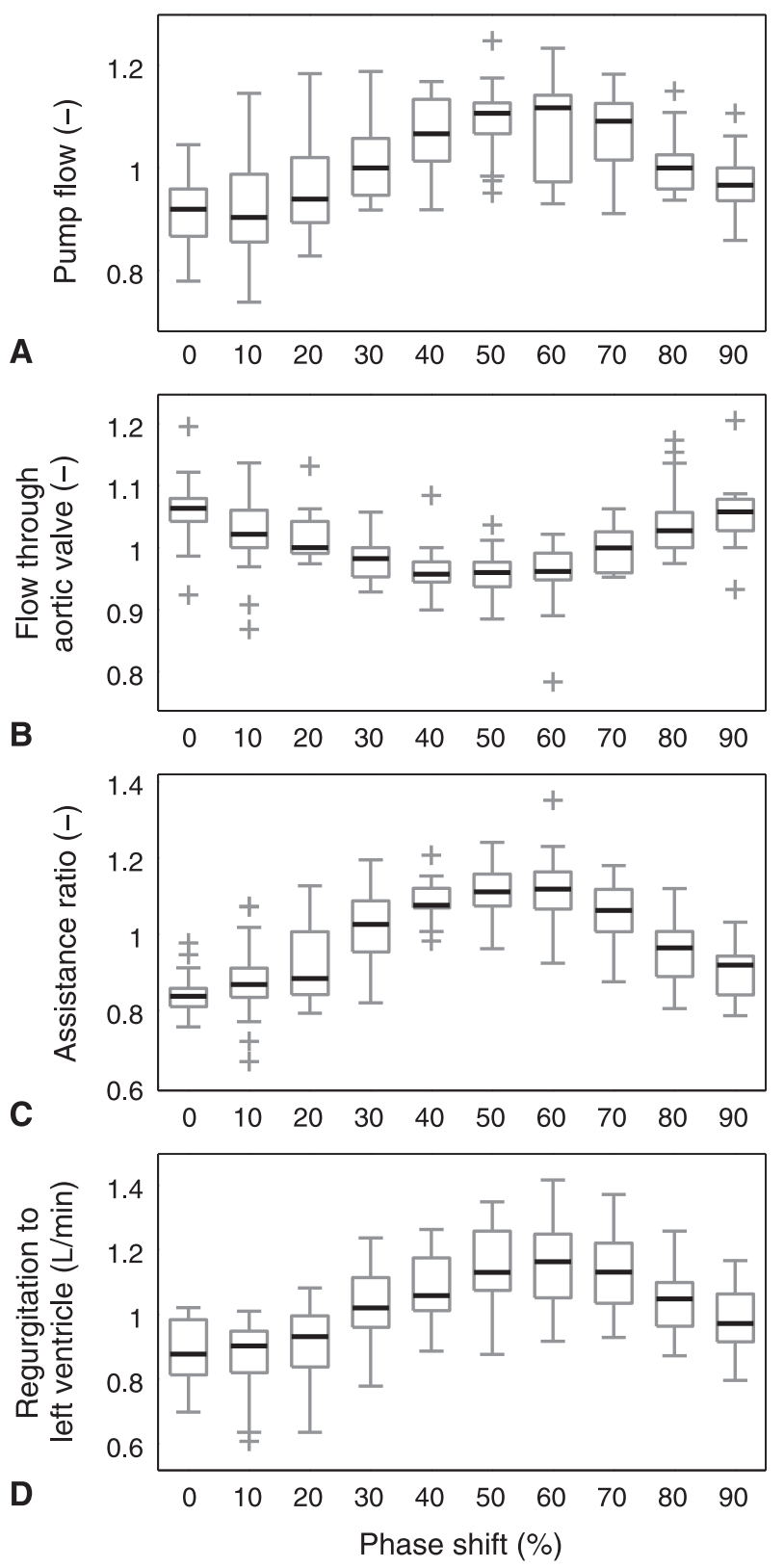

FIGURE 5. Boxplots of analyzed variables clustered according to their phase shift are shown (each box represents the median value and 25th and 75th percentiles; + denotes outliers). Panels A through D show pump flow, flow through the aortic valve, assistance ratio, and regurgitation to the left ventricle. Data points are normalized by the median values of the individual data sets because at baseline the pump is turned off.

with the potential for recovery because they lead to overload of the ventricle.

Furthermore, another potential practical benefit can be derived from phase shift control: In the potential setting of weaning the ventricular assistance during recovery, the load can be adjusted to a level equal to baseline, which means that the LV is performing the same amount of work as if the VAD would not be present. This can also be used to intermittently assess LV contractility and contractile reserve safely, whereas current methods require that the level of support is reduced or the pump is turned off, thereby increasing the risk of thrombus formation.

To resume the potential clinical implications of our findings, it can be concluded that pulsatile VADs with ventriculo-aortic cannulation and in synchronized mode should be operated at a phase shift of approximately $10 \%$ to benefit from the reduced LV stroke work and regurgitation, limited wall stress, reduced end-diastolic volume, and reduced mean left atrial pressure.

The main limitation of the data presented is that measurements were performed in healthy animals. Nevertheless, the results presented show a repeatable behavior over several animals even with strongly varying baseline hemodynamic conditions. The conclusions of this study may not be directly applicable to the case of heart failure and need further research. Furthermore, in a real clinical setting, arrhythmias and changing hemodynamic conditions would directly alter the effects of phase shift control or even make this tool useless. As an alternative to changing phase shift, a reloading of the supported LV could be achieved by a reduction of the stroke volume as proposed by Slaughter and colleagues. ${ }^{11}$

In clinical practice, no synchrony between the LV and the pulsatile VAD occurs ${ }^{13,14}$ because ECG synchronization was abandoned. This mode was not used because no satisfactory solution regarding arrhythmia handling was available and mobility of the patients was limited because of ECG monitors and wiring. Phase shift varies from beat to beat, permitting hemodynamically undesirable situations in accordance with the results presented. Studies with short phases of device deactivation and with the pulsatile VAD running have been analyzed. ${ }^{14,25}$ It can be speculated that alternately unloading and overloading the LV in terms of stroke work adversely affects a potential reverse remodeling process.

\section{CONCLUSIONS}

The use of ECG synchronization in pulsatile LVADs might add substantial benefits in the treatment of this patient group. Our findings suggest that phase shift could be used as a parameter to minimize the load of the heart and to avoid overloading situations. We also demonstrated that phase shift could serve for a gradual increase of the stroke work and thereby fine adaptation of the LV load. This method may be important for strategies aiming at myocardial recovery.

The authors thank the staff from the Surgical Research Unit, Department of Clinical Research, University of Bern, and Kathrin Maurer from the Department of Veterinary Anesthesia, University of Bern, for assistance with the experiments; Brigitta Gahl from the Department of Cardiovascular Surgery, Inselspital Bern, for statistics advice; and Bitmedical $\mathrm{GmbH}$ and Medtronic Switzerland for providing cannulas and cell saver disposables. 


\section{References}

1. Frazier O, Rose EA, Oz MC, Dembitsky W, McCarthy P, Radovancevic B, et al. Multicenter clinical evaluation of the HeartMate vented electric left ventricular assist system in patients awaiting heart transplantation. J Thorac Cardiovasc Surg. 2001;122:1186-95.

2. Frazier OH, Rose EA, McCarthy P, Burton NA, Tector A, Levin H, et al. Improved mortality and rehabilitation of transplant candidates treated with a long-term implantable left ventricular assist system. Ann Surg. 1995;222: 327-38.

3. Frazier OH, Myers TJ, Gregoric ID, Khan T, Delgado R, Croitoru M, et al. Initial clinical experience with the Jarvik 2000 implantable axial-flow left ventricular assist system. Circulation. 2002;105:2855-60.

4. Griffith BP, Kormos RL, Borovetz HS, Litwak K, Antaki JF, Poirier VL, et al. HeartMate II left ventricular assist system: From concept to first clinical use. Ann Thorac Surg. 2001;71:S116-20.

5. Klotz S, Deng MC, Stypmann J, Roetker J, Wilhelm MJ, Hammel D, et al. Left ventricular pressure and volume unloading during pulsatile versus nonpulsatile left ventricular assist device support. Ann Thorac Surg. 2004;77:143-50.

6. Letsou GV, Pate TD, Gohean JR, Kurusz M, Longoria RG, Kaiser L, et al. Improved left ventricular unloading and circulatory support with synchronized pulsatile left ventricular assistance compared with continuous-flow left ventricular assistance in an acute porcine left ventricular failure model. J Thorac Cardiovasc Surg. 2010;140:1181-8.

7. Kato TS, Chokshi A, Singh P, Khawaja T, Cheema F, Akashi H, et al. Effects of continuous-flow versus pulsatile-flow left ventricular assist devices on myocardial unloading and remodeling. Circ Heart Fail. 2011;4:546-53.

8. Crow S, John R, Boyle A, Shumway S, Liao K, Colvin-Adams M, et al. Gastrointestinal bleeding rates in recipients of nonpulsatile and pulsatile left ventricular assist devices. J Thorac Cardiovasc Surg. 2009;137:208-15.

9. Madigan JD, Barbone A, Choudhri AF, Morales DLS, Cai B, Oz MC, et al. Time course of reverse remodeling of the left ventricle during support with a left ventricular assist device. J Thorac Cardiovasc Surg. 2001;121:902-8.

10. Maybaum S, Epstein S, Beniaminovitz A, Tullio MD, Oz M, Bergmann SR, et al. Partial loading of the left ventricle during mechanical assist device support is associated with improved myocardial function, blood flow and metabolism and increased exercise capacity. J Heart Lung Transplant. 2002;21: 446-54.

11. Slaughter MS, Sobieski MA, Koenig SC, Pappas PS, Tatooles AJ, Silver MA. Left ventricular assist device weaning: Hemodynamic response and relationship to stroke volume and rate reduction protocols. ASAIO J. 2006;52:228-33.
12. Farrar DJ, Compton PG, Lawson JH, Hershon JJ, Hill JD. Control modes of a clinical ventricular assist device. IEEE Eng Med Biol Mag. 1986;5:19-25.

13. Maybaum S, Williams M, Barbone A, Levin H, Oz M, Mancini D. Assessment of synchrony relationships between the native left ventricle and the HeartMate left ventricular assist device. J Heart Lung Transplant. 2002;21:509-15.

14. Dalby MCD, Banner NR, Tansley P, Grieve LA, Partridge J, Yacoub MH. Left ventricular function during support with an asynchronous pulsatile left ventricular assist device. J Heart Lung Transplant. 2003;22:292-300.

15. Heredero A, Perez-Caballero R, Otero J, Rodriguez-Martinez D, QuintanaVillamandos B, Rodriguez-Bernal G, et al. Synchrony relationships between the left ventricle and a left ventricular assist device: an experimental study in pigs. Int J Artif Organs. 2012;35:272-8.

16. Nakamura T, Hayashi K, Seki J, Nakatani T, Noda H, Takano H, et al. Effect of drive mode of left ventricular assist device on the left ventricular mechanics. Artif Organs. 1988;12:56-66.

17. Pan J, Tompkins WJ. A real-time QRS detection algorithm. IEEE Trans Biomed Eng. 1985;32:230-6.

18. Wei CL, Valvano JW, Feldman MD, Pearce JA. Nonlinear conductance-volume relationship for murine conductance catheter measurement system. IEEE Trans Biomed Eng. 2005;52:1654-61.

19. Arts T, Bovendeerd PHM, Prinzen FW, Reneman RS. Relation between left ventricular cavity pressure and volume and systolic fiber stress and strain in the wall. Biophys J. 1991;59:93-102.

20. Yoshizawa M, Takeda H, Watanabe T, Miura M, Yambe T, Katahira Y, et al. An automatic control algorithm for the optimal driving of the ventricular-assist device. IEEE Trans Biomed Eng. 1992;39:243-52.

21. Drzewiecki G, Pilla J, Welkowitz W. Design and control of the atrio-aortic lef ventricular assist device based on $\mathrm{O}_{2}$ consumption. IEEE Trans Biomed Eng. 1990;37:128-37.

22. Platt KL, Moore TW, Barnea O, Dubin SE, Jaron D. Performance optimization of left ventricular assistance. A computer model study. ASAIO J. 1993;39:29-38.

23. Kawaguchi O, Pae WE, Daily WB, Sapirstein JS, Pierce WS. Left ventricular mechanoenergetics during asynchronous left atrial to aortic bypass. J Thorac Cardiovasc Surg. 1995;110:793-9.

24. Moazami N, Argenziano M, Kohomoto T, Yazdani S, Rose EA, Burkhoff D, et al. Inflow valve regurgitation during left ventricular assist device support may interfere with reverse ventricular remodeling. Ann Thorac Surg. 1998;65:628-31.

25. Vandenberghe S, Segers P, Steendijk P, Meyns B, Dion RAE, Antaki JF, et al Modeling ventricular function during cardiac assist: does time-varying elastance work? ASAIO J. 2006;52:4-8. 